\title{
Disability and quality of life in Charcot-Marie-Tooth disease type 1
}

\author{
G Pfeiffer, E M Wicklein, T Ratusinski, L Schmitt, K Kunze
}

\begin{abstract}
Objectives-Charcot-Marie-Tooth disease type I (CMT1) is a hereditary sensorimotor neuropathy causing variable degrees of handicap. The risk for relevant disability in respect to genetic counselling is unknown. An attempt was made to define it.

Methods-Disability and ambulation of 50 patients with CMT1 were scored by the Hauser ambulation index score and the Rankin scale. Rankin score 2 was subdivided into 2 a (independent without relevant slowness) and $2 \mathrm{~b}$ (independent, though at the cost of excessive time consumption). The sickness impact profile was assessed and compared with patients 6 months after stroke who were without mental deficit. To define at which degree sickness and disability become relevant for genetic counselling, the patients were asked whether they would refrain from childbearing if the children were at risk of inheriting a disease that caused as much disability as they experienced themselves. Results-Subdivision of Rankin score 2 was reliable and improved validity. High disability significantly predicted an attitude against childbearing (stepwise logistic regression) only with this subdivision. Thirty six per cent of the patients voted against childbearing. The cut off for relevant disability in respect to childbearing was a Rankin score higher than 2a, which was present in $44 \%$ of the patients. Psychosocial impact was comparable with patients with stroke and similar disability. Depression was present in $18 \%$ of the patients.

Conclusion-Subdivision of Rankin score 2 is recommended for the assessment of longstanding disability in neuromuscular disorders. Disability becomes relevant for the attitude towards childbearing as soon as everyday activities become markedly slow (Rankin score 2 b). Relevant disability occurred in $44 \%$ of the patients. Emotional stress in CMT is similar to that of patients with stroke and comparable disability.

(F Neurol Neurosurg Psychiatry 2001;70:548-550)
\end{abstract}

Keywords: Charcot-Marie-Tooth disease type 1; hereditary neuropathy; genetic counselling; Rankin scale; sickness impact profile
Prenatal diagnosis for Charcot-Marie-Tooth disease type 1 (CMT1) is now available. For adequate counselling, we have to know more about the impact of the disease. Information about the inheritance risk may be sufficient in uniformly severe disorders such as Duchenne muscular dystrophy. Even then, prospective parents ask for the consequences of the disease. ${ }^{1}$ Charcot-Marie-Tooth disease type 1 has been designated as a relatively mild disease. ${ }^{2}$ Others say that $20 \%$ of the patients are seriously handicapped. ${ }^{3}$ This information is too contradictory. Disability has not yet been properly assessed in CMT1. Use of the neurological disability score ${ }^{45}$ measures impairment rather than disability. Only 60 of 104 patients with CMT were able to assign themselves a score between 0 (no effect) and 10 (every activity impaired). ${ }^{6}$ Other studies $^{7}{ }^{8}$ did not consider the arms, which are often involved in CMT $1 .{ }^{9}$ To our surprise, the Rankin scale, ${ }^{10}$ a standard measure of disability in stroke, mutifocal motor neuropathy, and CIDP, has not yet been used in CMT1.

Our patients often realised their disability only after queries about professional and pastime activities, or when we witnessed their slow undressing. Timed motor activities in CMT1 are up to sixfold prolonged. ${ }^{11}$ Therefore we subclassified Rankin score 2 for independent but exceedingly slow patients. To illustrate the burden of CMT1, we also compared it with a more frequent and better known disease: stroke with predominantly physical disability.

We asked our patients whether they would advise against childbearing, if the prospective child would have similar disability. In this hypothetical setting, a vote against childbearing implies that the patient considers the disease to be so severe that it is better not to start a life with the disease. It thus informs about perceived quality of life, and defines "relevant disability" in regard to prenatal diagnosis.

\section{Patients and methods}

METHODS

The study was approved by the local ethics committee. A structured interview explored time consumption and disabilities for professional and pastime activities. The Rankin scale $^{10}$ was prospectively modified by subclassification of independent patients who required more than twice the time for everyday activities 


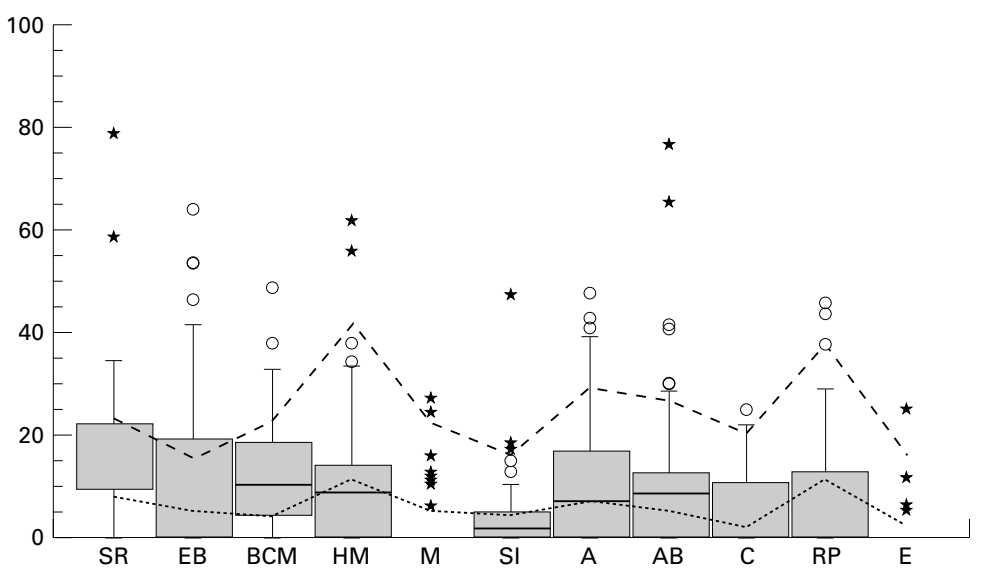

Figure 1 Sickness impact profile (SIP) of CMT1. The upper and lower boundaries of the boxplots of the SIP \% scores of our patients with CMT are the lower and upper quartiles. The bar indicates the median. The whiskers extend to the highest and lowest values that are less than one interquartile range from the box. Values beyond these limits are outliers (circles: within two interquartile ranges from the box; stars: more than two interquartile ranges from the box). The SIP scores are compared with the means of a group of 132 elderly controls (dotted line) and a group of 441 patients 6 months after a stroke (broken line) taken from the study of de Haan et al ${ }^{19}(S R=$ Sleep and rest; EB=emotional behaviour; $B C M=$ body care and movement; $H M=$ household management; $M=$ mobility; $S I=$ social interaction; $A=$ ambulation; $A B=$ alertness behaviour; $C=$ communication; $R P=$ recreation and pastime; $E=$ eating; the first three categories are combined to the physical dimension score and the next four categories, to the psychosocial dimension score.).

Table 1 Influence of different factors on the attitude towards childbearing

\begin{tabular}{llll}
\hline & $\begin{array}{l}\text { 50 patients with CMT (demyelination and (hereditary or confirmed } \\
\text { mutation)) }\end{array}$ & Univariate & $\begin{array}{l}\text { Logistic } \\
\text { regression }\end{array}$ \\
\hline 1 & Age (range 17-76 y; mean 47 y) & 0.35 & \\
2 & Sex (male 19; female 31) & 0.98 & 0.03 \\
3 & Affected family members of previous generation (35 patients) & 0.15 & 0.03 \\
4 & Presence of children (24 patients) & 0.02 & 0.04 \\
$5^{\star}$ & Rankin score (1, 4 patients; 2a, 24 patients; 2b nine paients; 3, 12 & 0.01 & \\
& paients; 4 one patient) & 0.28 & \\
6 & Deterioration within the past 5 years (27 patients) & 0.18 & \\
7 & SIP: physical dimension & 0.83 & \\
8 & Neuropathy related pain or painful muscle cramps (34 patients) & 0.94 & \\
9 & SIP: psychosocial dimension & 0.13 & \\
10 & Depression (eight patients) & 0.30 & \\
11 & Regular use of healthcare institutions (16 patients) & 0.09 & \\
12 & Use of orthopaedic devices (26 patients) & & \\
13 & Attitude towards childbearing (pro 32 patients; contra 18 patients) & & \\
\hline
\end{tabular}

^For calculations values of 2 and 2.5 were used for Rankin grades $2 \mathrm{a}$ and $2 \mathrm{~b}$. SIP=Sickness impact profile.

than healthy companions or colleagues (Rankin score 2b). Two independent observers ( $\mathrm{E} \mathrm{W}$ and $\mathrm{T} \mathrm{R}$ ) concurred in this respect in 31 of the 33 patients. Ambulation was scored according to Hauser et al. ${ }^{12}$ Disease impact was measured by a German translation of the sickness impact profile (SIP) validated for musculoskeletal disorders. ${ }^{13}{ }^{14}$ The SIP provides 141 questions addressing problems in 12 categories of everyday functions (fig 1). Affirmative answers are weighted and summed up for every category. The scores are reported as the percentage of the maximum impact. We used the self rating depression scale (SDS). ${ }^{15}$

Twelve biographical, physical, and psychosocial variables were related to the attitude towards childbearing (table 1 ) by $\chi^{2}$ (nominal items) or Mann-Whitney tests (ranked scales) and by logistic regression (SPSS for Windows). Seven patients were excluded from multivariate analysis because of missing values. Variables were selected stepwise backwards, using the conditional statistic. ${ }^{16}$ Logistic regression models are linear combinations of the independent variables and yield $\mathrm{Z}$ scores which allow the calculation of how probable it is that a given person has a positive or negative attitude towards childbearing. Comparison with the actual attitude of the patients shows how good the model explains the attitude.

\section{PATIENTS}

Fifty patients met clinical criteria for $\mathrm{CMT} 1^{17}$; 35 index patients and 15 affected relatives did not differ by Rankin score $(\mathrm{p}=0.32)$. Forty six patients had a positive family history. The four sporadic patients had a duplication on chromosome $17 \mathrm{p} 11.2$, which was shown by Southern blot hybridisation in 29 patients. Neither patients nor partners were pregnant. Twelve patients had unaffected children. At least one child had CMT1 in 12 families. The SIP was determined at the 6 month follow up of 23 successive patients with stroke without aphasia or mental deficit according to the mini mental state examination.

\section{Results}

Disability neither depended on age $(p=0.60)$, nor on the presence of a duplication. Gait was normal in five patients (Hauser ambulation index score (HAS) 0 or 1). Twenty seven patients with abnormal gait managed 8 metres in less than 10 seconds (HAS 2). Fifteen patients walked without support but required between 10 and 20 seconds for 8 metres (HAS 3). Manual dexterity was impaired in 26 patients. Twenty seven patients had deteriorated during the past 5 years. The disease influenced choice of profession or necessitated retraining or early retirement in $36 \%$ of the patients. Related to the Rankin score $(p=0.02)$, the disease interfered with professional life in $58 \%$ of the patients. The 35 patients with restricted pastime activities had higher physical $(p=0.013)$ and psychosocial $(p=0.025)$ SIP scores. Thirty four patients complained of neuropathy related pain or painful muscle cramps. Seventy six per cent of the patients consulted doctors because of neuropathy related complaints; $38 \%$ of the patients regularly received physical therapy; 52\% used orthopaedic devices. The SDS $^{18}$ indicated depression in eight of 50 patients, unrelated to disability $(\mathrm{p}=0.48)$.

The median SIP scores were similar to means of elderly control subjects ${ }^{19}$ except for "sleep and rest" and "body care and movement", which exceeded the control means in more than $75 \%$ of the patients with CMT (lower boundary of the boxes in the figure). Neither the Rankin scores nor the SIP scores "emotional behaviour", "body care and movement", "ambulation", and "eating" differed between patients with CMT1 and those with stroke. The other SIP scores were significantly lower in CMT1. The 42 SIP outliers (fig 1) indicating exceptionally high impact were from 18 patients.

Thirty two patients favoured childbearing. Low Rankin score $(p=0.014)$ and the fact that the patient already had children $(p=0.023)$ predicted a positive attitude towards child bearing. No patient with Rankin score 1 and a quarter of the patients with Rankin score $2 \mathrm{a}$ discouraged childbearing, whereas five of nine 
patients with Rankin scores $2 \mathrm{~b}$ and seven of 12 patients with Rankin score 3 did so. The full regression model with all variables predicted the attitude towards child bearing correctly in $81.4 \%$ of the patients. The final model:

$Z=1.54-1.43$ * $($ Rankin score $)+2.02^{\star}$ (presence of children) $+2.25^{\star}$ (presence of affected family members from the previous generation)

predicted the attitude correctly in $76.7 \%$ of the patients. The different signs for "Rankin score", and "presence of children" and "presence of affected family members" indicate effects in opposite directions: high disability predicts a vote against childbearing, whereas presence of own children or affected family members favours childbearing. Various backward selection starting from subsets of the full model selected the same final model. After reclassification of Rankin scores $2 \mathrm{a}$ and $2 \mathrm{~b}$ into 2 , disability dropped from the logistic regression model.

\section{Discussion}

We expected higher emotional stress in patients with stroke because they have less time to develop successful coping. However, emotional stress was comparable in both diseases. Patients with CMT scored unexpectedly high in two SIP domains: "sleep and rest" and "alertness behaviour", due to frequent affirmations to "I sit during the day", "I lie down more often during the day in order to rest", "I have minor accidents", or "I react slowly to things that are said or done". These statements infer something different from that intended by the SIP designers, but illustrate typical problems of our patients, who behaved clumsily, which may provoke teasing already at school age, stamping them early as outsiders who, when thriving at normal professional achievements, will have to fight permanently because they are slow in important activities. ${ }^{11}$ Higher rest requirements and pain are additional burdens. The high percentage of patients seeking medical help ${ }^{15}$ also falsifies the idea that impairment is hard to perceive in CMT1. ${ }^{3}$ The worst case scenario for prospective parents deciding about prenatal diagnosis $^{20}$ includes the facts that CMT1 affects choice of profession, severely slows important activities, requires help in everyday life, and causes emotional distress similar to other chronic conditions.

The split between irrelevant and relevant disability passed through Rankin grade 2: disability dropped from stepwise regression after reclassification of Rankin scores $2 \mathrm{a}$ and $2 \mathrm{~b}$ into 2 . We suggest a division of Rankin grade 2 for CMT and other diseases with longstanding physical disability.

Disability was similar to that found in other studies. Forty of 119 patients with CMT1A were unable to $\operatorname{run}^{8}: 56 \%$ of our patients had similar walking difficulties (HAS $>2$ ). Another study $^{7}$ found a similar degree of ambulation impairment (walking obviously impaired, running impossible) in $42 \%$ of their patients. Relevant disability (Rankin score $>2 \mathrm{a}$ ) was present in $44 \%$ of our patients. The vote against childbearing $(36 \%)$ underestimates the prevalence of relevant disability because family factors opposed its effect, as shown by logistic regression. Therefore, the inheritance risk in mathematical decision models for prenatal diagnosis should be weighted by 0.44 , corresponding to the percentage of patients with Rankin grade above $2 \mathrm{a}$.

EMW was supported by the Marta- und Erik-Karberg foundation.

1 Beeson D, Golbus MS. Decision making: whether or not to have prenatal diagnosis and abortion for X-linked conditions. Am f Med Genet 1985;20:107-14.

2 de Jonghe P, Timmerman V, Nelis E, et al. Charcot-MarieTooth disease and related peripheral neuropathies. $f$ Peripher Nerv Syst 1997;2:370-87.

3 Reilly MM. Genetically determined neuropathies. $\mathcal{F}$ Neurol 1998;245:6-13.

4 Dyck PJ, Karnes JL, Lambert EH. Longitudinal study of neuropathic deficits and nerve conduction abnormalities in hereditary motor and sensory neuropathy type 1 . Neurology 1989;39:1302-8

5 McMillan JC, Harper PS. The Charcot-Marie-Tooth syndrome: clinical aspects from a population study in South Wales, UK. Clin Genet 1994;45:128-34.

6 McMillan JC, Harper PS. The Charcot-Marie-Tooth syndrome: perceptions of disability and projected use of DNA diagnostic tests. Clin Genet 1992;42:161-3.

7 Holmberg BH. Charcot-Marie-Tooth disease in northern Sweden: an epidemiological and clinical study. Acta Neurol Scand 1993;87:416-22.

8 Birouk N, Gouider R, Le Guern E, et al. Charcot-MarieTooth disease type $1 \mathrm{~A}$ with $17 \mathrm{p} 11.2$ duplication. Clinical and electrophysiological phenotype study and factors influencing disease severity in 119 cases. Brain 1997;120:81323.

9 Thomas PK, Marques W, Davis MB, et al. The phenotypic manifestations of chromosome 17p11.2 duplication. Brain 1997;120:465-78.

10 Van Swieten JC, Koudstaal PJ, Visser MC, et al. Interobserver agreement for the assessment of handicap in stroke patients. Stroke 1988;19:604-7.

11 Carter GT, Abresh RT, Fowler WM, et al. Profiles of neuromuscular diseases. Hereditary motor and sensory neuropathy, types I and II. Am 7 Phys Med Rehabil 1995;749(suppl):S140-9.

12 Hauser SL, Dawson DM, Lehrich JR, et al. Intensive immunosuppression in progressive multiple sclerosis. $N$ Engl f Med 1983;308:173-80.

13 Bergner M, Bobitt R A, Pollard W E, et al. The sickness impact profile. Validation of a health status measure. Med Care 1976;14:57-67.

14 Kessler S. Testtheoretische Prüfung der deutschen Fassung des Sickness Impact Profile bei Erkrankungen des Stütz- und Bewegungsapparates [dissertation]. Ulm, 1991.

15 Wicklein EM, Pfeiffer G, Ratusinski T, et al. CharcotMarie-Tooth syndrom Typ I. Behinderung und Management. Nervenarzt 1997. 68:358-62.

16 Norusis MJ. SPSS for Windows: advanced statistics, release 6.0. Chicago: SPSS, 1993.

17 de Visser M, v Broeckhoven C, Nelis E. Hereditary motor and sensory neuropathy or Charcot-Marie- Tooth disease types $1 \mathrm{~A}$ and B In: Emery AEH. ed. Diagnostic criteria for neuromuscular disorders. Oxford: Pergamon Press, 1994, $49-52$.

18 Zung WWK, Richards CB. Self-rating depression scale. Arch Gen Psychiatry 1965;12:63-70.

19 de Haan RJ, Limburg M, van der Meulen JHP, et al. Quality of life after stroke. Impact of stroke type and lesion location. Stroke 1995;26:402-8.

20 Lipman-Hand A, Fraser FC. Genetic counseling: the postcounseling period: I. parents' perceptions of uncertainty. Am F Med Genet 1979;4:51-71. 